\title{
Insight into eco-friendly fabrication of silver nanoparticles by Pseudomonas aeruginosa and its potential impacts
}

\author{
Jafar Ali1, 2,3 Naeem Ali ${ }^{3}$, Syed Umair Ullah Jamil ${ }^{4}$, Hassan Waseem ${ }^{5}$, \\ Kifayatullah Khan ${ }^{6,7}$ and Gang Pan*1,8 \\ ${ }^{1}$ Laboratory of Environmental Nanomaterials, Research Center for Eco-environmental Sciences, Chinese Academy \\ of Sciences, 18 Shuangqing Road, Beijing 100085, PR China \\ ${ }^{2}$ University of Chinese Academy of Sciences, Beijing 100049, PR China \\ ${ }^{3}$ Department of Microbiology, Quaid-i-Azam University Islamabad, Pakistan \\ ${ }^{4}$ Department of Earth and Environmental Sciences, Bahria University Islamabad, Pakistan \\ ${ }^{5}$ Department of Civil and Environmental Engineering, Michigan State University, East Lansing, MI 48824, USA \\ ${ }^{6}$ Department of Environmental and Conservation Sciences, University of Swat, Swat 19130, Pakistan \\ ${ }^{7}$ State Key Lab of Urban and Regional Ecology, Research Center for Eco-Environmental Sciences, Chinese Academy \\ of Sciences, Beijing 100085, China \\ ${ }^{8}$ School of Animal, Rural and Environmental Sciences, Nottingham Trent University,Brackenhurst Campus, NG25 \\ OQF, UK \\ *Corresponding authors: \\ ${ }^{1}$ Laboratory of Environmental Nanomaterials, Research Center for Eco-environmental Sciences, \\ Chinese Academy of Sciences, 18 Shuangqing Road, Beijing 100085, PR China \\ Gang Pan, Tel: 010-62949686 Fax : 010-62943436. Email: Gpan@rcees.ac.cn
}

\begin{abstract}
Although green synthes is of nanoparticles (NPs) has replaced conventional physicochemical methods owing to eco-friendly and cost effective nature but molecular mechanism is not known completely. Elucidation of the mechanism is needed to enhance the production of control size synthesis and for understanding the biomineralization process. Here we report the facile, extracellular biosynthesis of silver nanoparticles (AgNPs) by Pseudomonas aeruginosa JP1 through nitrate reductase mediated mechanism. $\mathrm{AgNO}_{3}$ was reduced to AgNPs by cell filtrate exposure. UV-visible spectrum of the reaction mixture depicted reduction of ionic silver $\left(\mathrm{Ag}^{+}\right)$to atomic silver $\left(\mathrm{Ag}^{0}\right)$ by a progressive upsurge in surface plasmon resonance (SPR) band range $435-450 \mathrm{~nm}$. X-ray diffraction analysis showed the $2 \theta$ values at $38.08^{\circ}, 44.52^{\circ}, 64.42^{\circ}$ and $77.44^{\circ}$ confirming the crystalline nature and mean diameter $[6.5-27.88 \mathrm{~nm}($ Ave $=13.44 \mathrm{~nm})]$ of AgNPs. Transmission electron microscopy analysis demonstrated the spherical AgNPs with size range 5-45 nm. Stabilizing proteins and rhamnolipids were recognized by Fourier transform infrared spectroscopy. Nitrate reductase was purified and characterized (molecular weight $65 \mathrm{kDa}$ and specific activity $=5.6 \mathrm{U} / \mathrm{mg}$ ). To probe the plausible mechanism purified enzyme was retreated with $\mathrm{AgNO}_{3}$. Characteristic SPR bands range $(435-450 \mathrm{~nm})$ and Particle-induced x-ray emission results also confirmed the synthesis of AgNPs (59679.5 ppm) in solution. These results demonstrated that, nitrate reductase as a principal reducing agent in the mechanistic pathway of AgNPs synthesis, which leads to the understanding of metal transformation and biomineralization processes for controlling the biogeochemical cycles of silver and other heavy metals.
\end{abstract}

Key words: Eco-friendly, Pseudomonas aeruginosa, Silver nanoparticles, Nitrate reductase, Biomineralization 


\section{Introduction}

Biological synthesis of nanoscale materials is a limelight of modern nanotechnology. Silver nanoparticles (AgNPs) have garnered much attention due to the wide range of applications in catalysis ${ }^{1}$, membrane bioreactors ${ }^{2}$, DNA sequencing ${ }^{3}$ and cancer treatment ${ }^{4}$. Several physical and chemical strategies have been employed for the production of nanoparticles (NPs) ${ }^{5}$. Conventional synthesis procedures are becoming obsolete due to the high $\operatorname{cost}^{6}$, hazardous nature ${ }^{7}$, and low yield ${ }^{8}$. Since there is growing need to explore the alternative synthesis protocols which are facile, eco-friendly and cost effective. As inspired by the bioreduction of silver ion $\left(\mathrm{Ag}^{+}\right)$by Pseudomonas stutzeri ${ }^{9}$, green synthesis of AgNPs using microorganisms has become a hot topic ${ }^{10}$. Extracellular bacteriogenic synthesis is preferred due to rapid growth and simplified downstream processing. Although biogenic synthesis has been also demonstrated by fungus ${ }^{11}$, plants extracts like starch $^{12}$ and enzymes ${ }^{13}$ but molecular mechanism yet to be elucidated for enhanced and controlled size synthesis ${ }^{14}$.

Complete understanding of the synthesis pathway will be helpful in bio-mineralization and biotransformation of heavy metals. Basic insights of enzymes-metal interactions are also essential to overcome bottlenecks associated with bioremediation strategies ${ }^{15}$. Several studies have suggested the involvement of nitrate reductase in bio-reduction of metal ions ${ }^{16}$. Fungal mediated invitro synthesis of AgNPs was reported from Fusarium oxysporum based $\alpha-\mathrm{NADPH}$ dependent nitrate reductase acted as electron shuttle ${ }^{17}$. Moreover, biological reduction of $\mathrm{Ag}^{+}$was partially inhibited by piperitone for enterobacteria emphasizing the critical role of specific enzyme ${ }^{18}$. Remarkably periplasmic nitrate reductase (NapC) have been linked to the intracellular AgNPs formation by metal reducing E.coli ${ }^{18}$. Jain et al speculated the possible mechanism of extracellular AgNPs synthesis in Aspergillus flavus NJP08 ${ }^{19}$.

Another study demonstrated superoxide mediated synthesis of AgNPs by a fungus and indirectly linked nitrate reductase participation ${ }^{20}$. Recently AgNPs synthesis has been investigated with immobilized NADH-dependent nitrate reductase hence substantiating the enzymatic (Nitrate reductase) reduction in underlying mechanism of AgNPs synthesis ${ }^{21}$. Numerous studies have purified the nitrate reductase as prime reducing agent but some additional evidence and validations are still needed to support the mechanistic theory. We assume that resynthesizing the AgNPs from purified enzyme may corroborate the proposed pathway. In our previous study nitrate reductase was probed as the principal reducing agent through positive correlation between enzymatic specific activity and AgNPs synthesis ${ }^{22}$.

In present study, efforts were directed to validate the enzymatic pathway of extracellular AgNPs synthesis. Pseudomonas aerogenosa JP1 isolated from a metal contaminated soil was used a source of 
nitrate reductase. Nitrate reductase activity of cell filtrate was evaluated for reduction of silver nitrate to AgNPs. Extracellularly synthesized AgNPs were demonstrated by UV-vis XRD, FTIR, TEM along with purification of nitrate reductase. Purified enzyme retreatment with $\mathrm{AgNO}_{3}$ resynthesized the AgNPs which symmetrically depicted the role of nitrate reductase in mechanistic pathway. Efficient metal transformation strategies will improve geo-microbiological processes in metal contaminated environments.

\section{Experimental}

\subsection{Biosynthesis of silver nanoparticles}

In this study, extracellular AgNPs were synthesized using the cell filtrate of a Pseudomonas aerogenosa JP1 isolated from the metal contaminated soil. Purified bacterial culture was aerobically cultivated in the slightly modified MGYP media containing glucose $10 \mathrm{~g} / \mathrm{L}$, peptone $5 \mathrm{~g} / \mathrm{L}$, malt extract $3 \mathrm{~g} / \mathrm{L}$ and yeast extract $3 \mathrm{~g} / \mathrm{L}$ for $100 \mathrm{~mL}$ growth medium at $37^{\circ} \mathrm{C}$ on a rotary shaker $(12 \mathrm{xg})$ for $24 \mathrm{~h}$. Cell free extract was obtained by harvesting the bacterial culture after centrifugation at $13416 \mathrm{x} g$ at $4{ }^{\circ} \mathrm{C}$ for $15 \mathrm{~min}$ (centrifuge Model H-251, Kokusan Co., Ltd., Tokyo, Japan). Equal volumes of the supernatant were mixed with the aqueous solution $(10 \mathrm{mM})$ of silver nitrate in Erlenmeyer flask (150 $\mathrm{mL})$ and incubated at $37^{\circ} \mathrm{C}$ in the rotary shaker $(12 \mathrm{x} \mathrm{g})$ for $8 \mathrm{~h}$. Subsequently, AgNPs were concentrated and washed with chilled ethanol to remove media components. Air dried AgNPs were subjected to further characterization.

\subsection{Characterization of silver nanoparticles}

Preliminary characterization for AgNPs was done by noticing the visible colour change. Enzymatic reduction of silver ions $\left(\mathrm{Ag}^{+}\right)$was also perceived by measuring the optical density of the reaction mixture via $\mathrm{UV}-\mathrm{V}$ is spectrophotometer (Agilent 8453) at different time intervals along with symmetrically noticing the visible colour change. X-ray diffraction (XRD) analysis was done to confirm crystalline nature and mean diameter. Silica powder-coated film of AgNPs were subjected to XRD analysis operating at $30 \mathrm{kV}, 20 \mathrm{~mA}$ with $\mathrm{CuK} \alpha$ radiation in a transmission mode, (X'pert PRO XRD, PANalytical BV, Almelo, and The Netherlands) A carbon-coated copper transmission electron microscopic (TEM) grid was prepared containing a film of AgNPs and examined by TEM at an accelerating voltage of $80 \mathrm{kV}$ (JEM-1010, JEOL Ltd, Tokyo, Japan). Fourier transform infrared spectroscopy (FTIR) is a sensitive technique to quantify the secondary structure of proteins through the resonance of non-centrosymmetric mode of vibrations ${ }^{23}$. To identify the capping molecule nature and interaction with metal NPs was analysed by FTIR machine (Model 200-VT, Perkin-Elmer, Shelton, CT). 


\subsection{Enzyme Characterization for Elucidation of molecular mechanism}

Enzyme characterization was done by protein precipitation of bacterial cell filtrate with $70 \%$ ammonium sulphate saturation. Protein precipitates were concentrated by centrifugation at $4830 \mathrm{x} \mathrm{g}$ and $4^{\circ} \mathrm{C}$ for 20 min (centrifuge Model H-251, Kokusan Co., Ltd., Tokyo, Japan). Crude protein pellet was suspended in phosphate buffer $(50 \mathrm{mM})$ with $\mathrm{pH} 7.4$ to estimate the protein content (Bradford assay) ${ }^{24}$ and nitrate reductase activity as described in literature ${ }^{25}$. Crude protein was further purified by size exclusion chromatography with Sephadex G-100 and phosphate buffer (pH 7.4). Size exclusion chromatography is useful technique to separate the protein molecules on size basis ${ }^{26}$. The molecular size of purified nitrate reductase was determined by SDS-PAGE and proteins were visualized by staining with Coomassie brilliant blue R-250.

Molecular Size was determined by comparison with standard protein marker (Bio-Rad, USA) 27. To Probe the molecular mechanism of NPs synthesis purified nitrate reductase was retreated with $\mathrm{AgNO}_{3}$ solution $(10 \mathrm{mM})$. Heat Inactivated purified enzyme was also given the same treatment as a control and test tubes were incubated at $37^{\circ} \mathrm{C}$ for $8 \mathrm{~h}$. The reaction mixture was characterized by UVvis spectroscopy and Particle induced $\mathrm{x}$-ray emission analysis (PIXE) after the visible colour change. Previously, PIXE analysis has been used to determine the elemental composition with minimal sample preparation and higher sensitivity ${ }^{28}$. For PIXE analysis pelletized samples were irradiated with the $3 \mathrm{MeV}$ proton beam from the 5MV Pelletron Tandem accelerator. The emitted x-rays were detected by a $30 \mathrm{~mm}^{2} \mathrm{Si}(\mathrm{Li})$ detector and energy resolution of $138 \mathrm{eV}$ (FWHM) at 5:9 keV of Mn. GUPIXWIN v 2.2.3 software ${ }^{29}$ was used to process the PIXE data.

\section{Results and discussion}

\subsection{UV-Vis spectrophotometric analysis}

Primary detection of AgNPs synthesis was done by the visible colour change from light yellow to dark brown (Figure.1A). The gradual colour change was the clear indication of $\mathrm{AgNO}_{3}$ reduction through a catalytic component present in cell filtrate (Figure 1A). The specific colour change was due to the excitation of Surface Plasmon Resonance (SPR) in the production of AgNPs. The spectra of AgNPs showed the strong absorption (SPR) in range of 435-450 nm (Figure 1B). Progressive colour change and corresponding increasing intensities of UV-vis spectra were perceived up to 8 hours. The high intensity of SPR band was probably due to increased concentration of AgNPs in reaction mixture ${ }^{30}$. Later on, there was no increase in SPR band indicating the completion of the synthesis reaction. Previously, biosynthesis of AgNPs has been reported by Bacillus sp CS 11 with SPR at $450 \mathrm{~nm}^{31}$. 


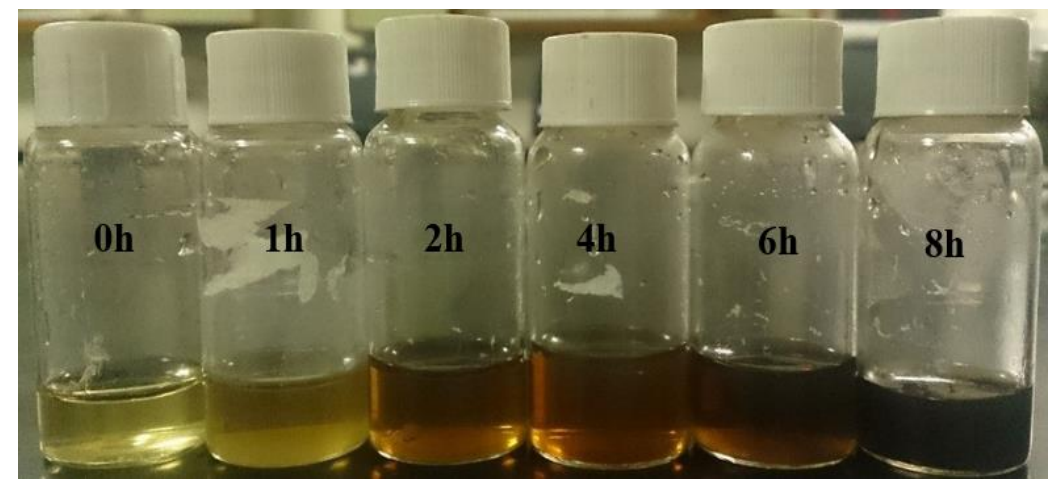

Figure. 1(A). Visible Colour change of the reaction mixture at diffe rent interval

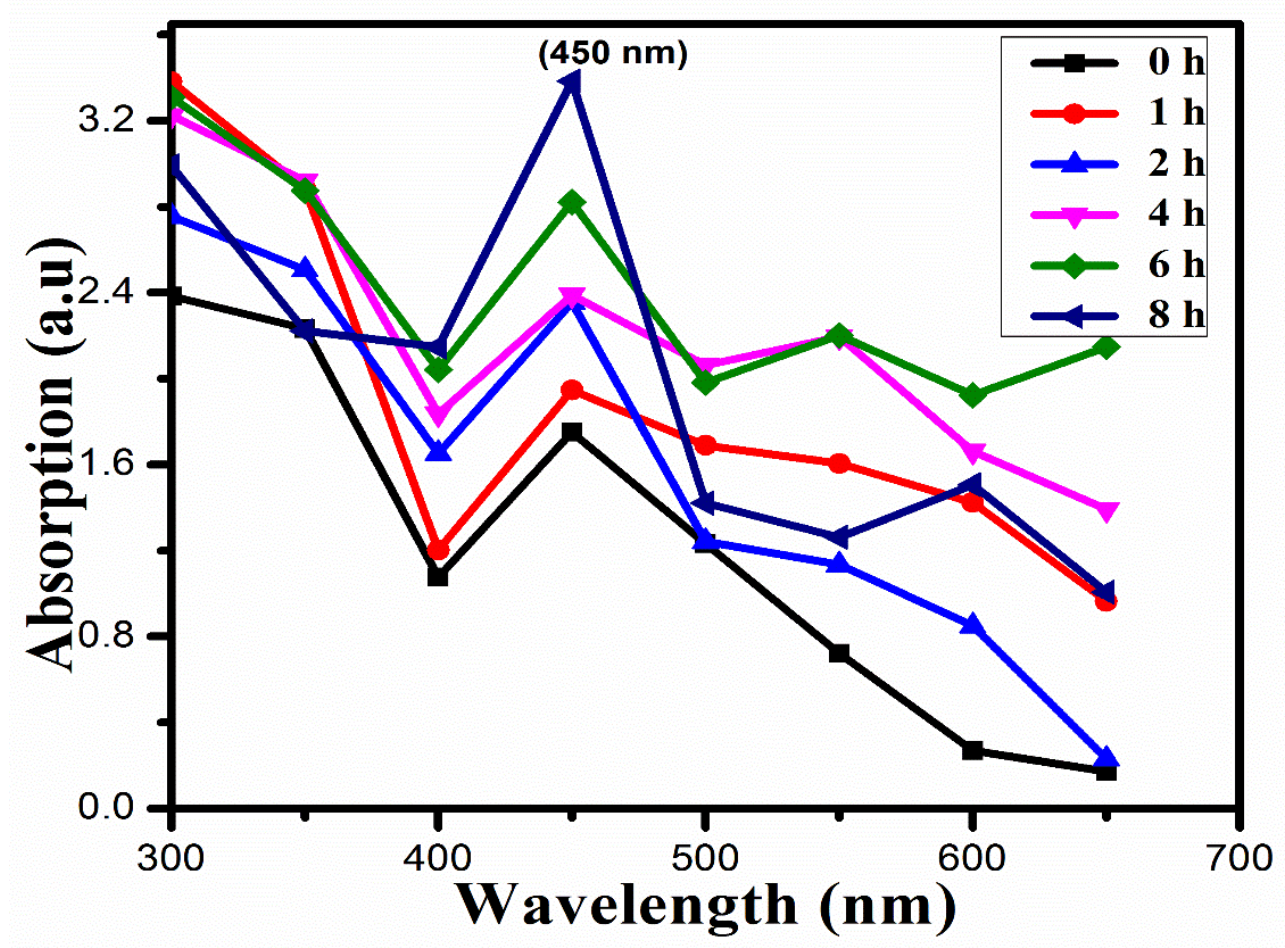

Figure. 1(B): UV-Visible absorption spectrum of AgNPs at diffe rent time intervals.

\section{2. $X$-ray diffraction $(X R D)$ analysis}

X-ray diffraction analysis was performed to determine the crystalline structure of AgNPs. Figure.3 illustrates the XRD pattern of biosynthesized AgNPs by using Pseudomonas aeruginosa JP1 extract. XRD pattern exhibited specific Bragg peaks at $2 \theta$ values of $38.08^{\circ}, 44.52^{\circ}, 64.42^{\circ}$ and $77.44^{\circ}$ which are indexed by hkl planes 111, 200, 220 and 311 of the face-centered cubic (fcc) crystal structure (JCPDS card no.04- 00783). XRD results clearly displayed that pure AgNPs were produced by enzymatic reduction (Figure 2A). Debye-Scherer equation was used to determine the size range of AgNPs 6.5-27.88nm with mean diameter 13.44nm (Figure 2B). Our result corroborates the previous 
findings of extracellular synthesis of AgNPs (13nm) by Pseudomonas aeruginosa ${ }^{32}$. Small insignific ant peaks were also observed in XRD pattern showing some organic impurities present in the reaction mixture $^{33}$.

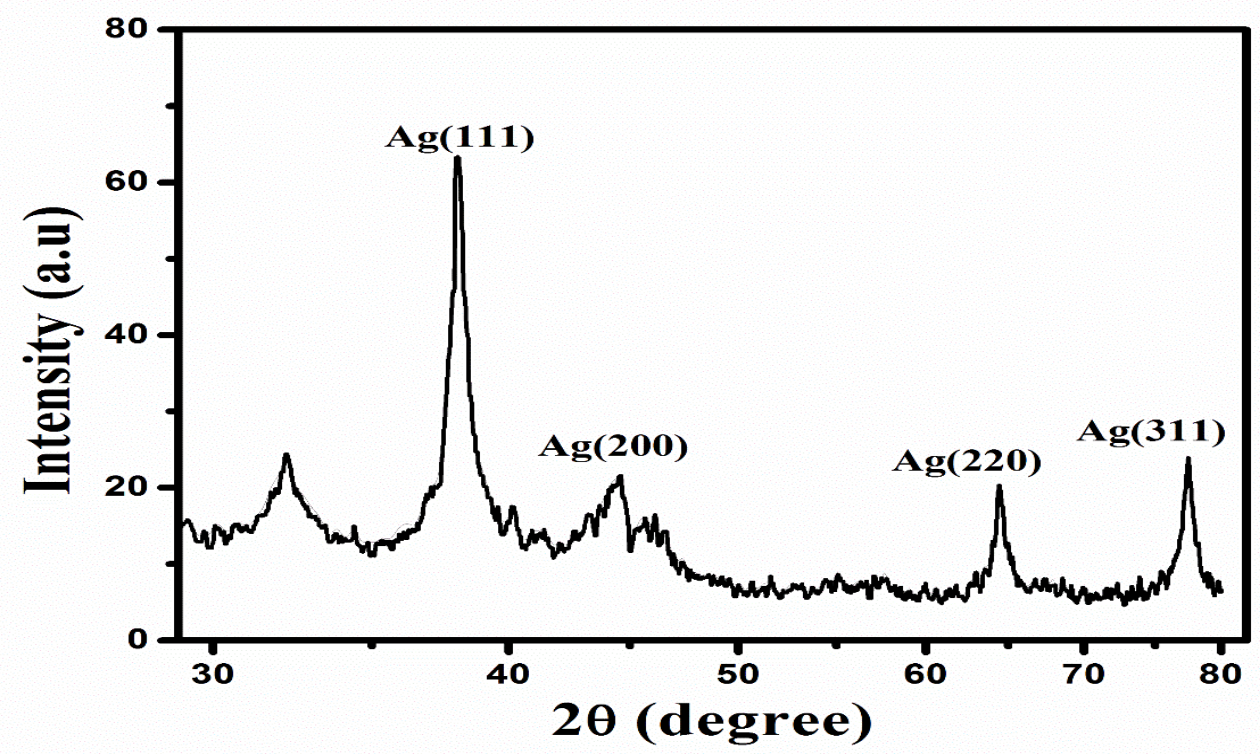

Figure 2(A): XRD Pattern of AgNPs synthesized by Pseudomonas aeruginosa JP1.

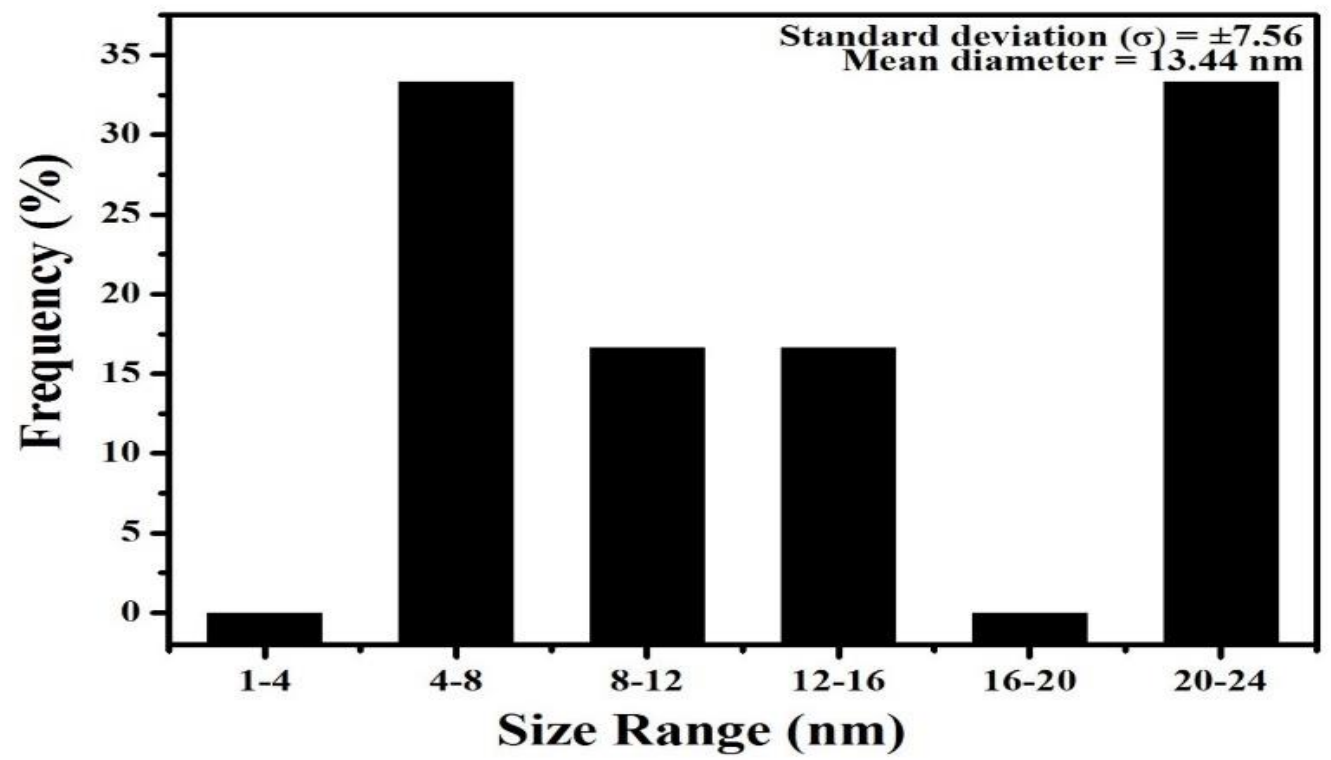

Figure.2 (B): Histogram showing the AgNPs Size distribution.

\subsection{Transmission Electron Microscopy (TEM)}

Transmission electron micrographs clearly showed synthesized distinct and spherical AgNPs (Figure. 3). TEM analysis provided the size distribution in range 5-45nm for AgNPs (figure. 3) which is in agreement with XRD results (Figure.2). Despite some aggregates majority of AgNPs was monodispersed 
and stabilized by capping molecules (Figure. 3). Monodispersity can be linked with capping proteins present on the surface of AgNPs. Zaki et al (2011) have reported similar results for AgNPs synthesis (15$50 \mathrm{~nm})$ by different bacterial isolate ${ }^{34}$.

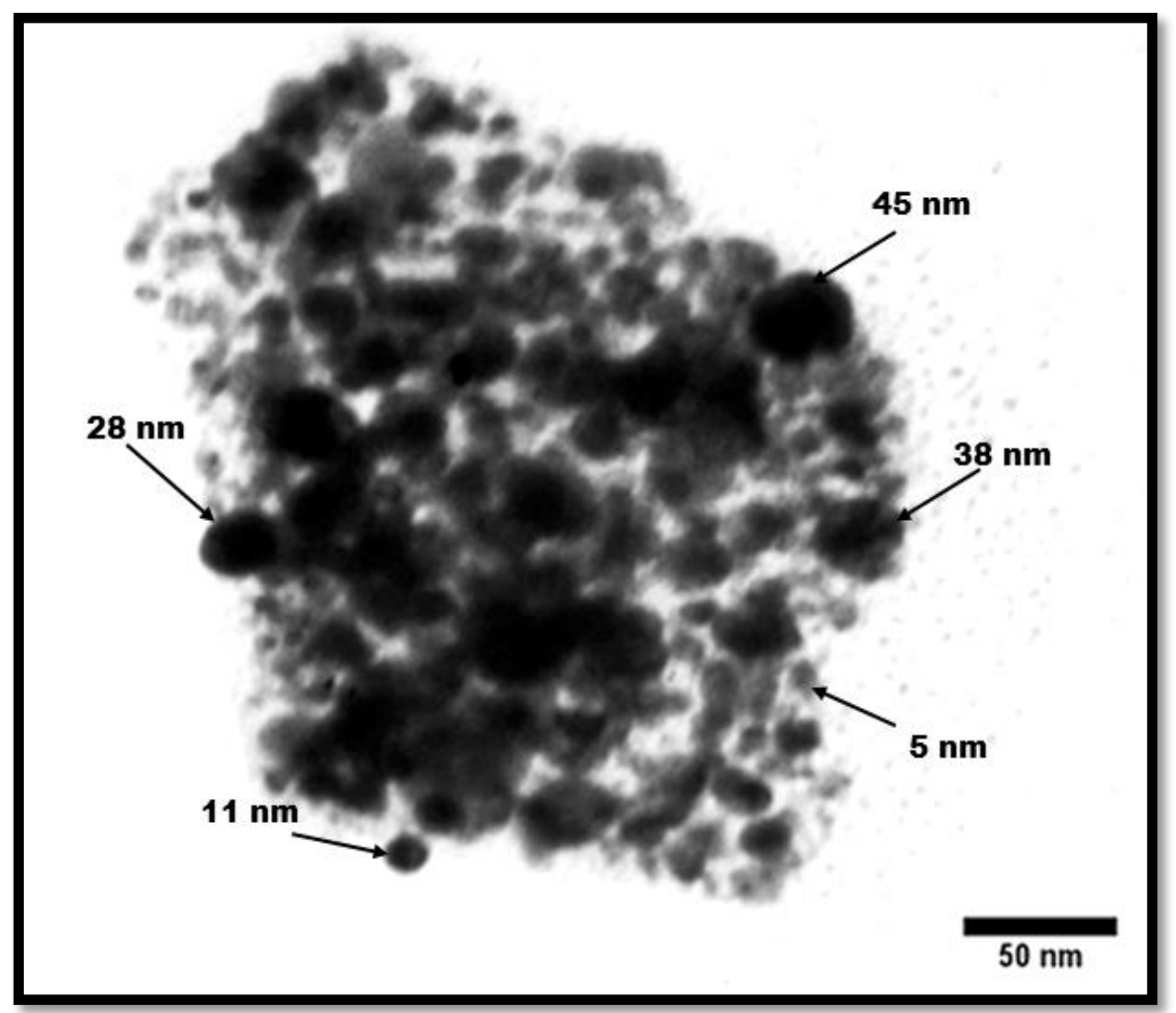

Figure. 3: TEM images of AgNPs synthesized by Pseudomonas aeruginosa JP1.

\subsection{Fourier Transform Infrared (FTIR) Spectroscopy}

Fourier transform infra-red (FTIR) analysis was performed to identify the nature of stabilizing molecules. FTIR spectrum showed the major absorption bands at 782, 1022,1055,1149,1368, 1599, 1632, 2869 and $2978 \mathrm{~cm}^{-1}$ (Figure.4). The band $782 \mathrm{~cm}^{-1}$ can be linked to $\mathrm{C}-\mathrm{H}$ group of proteins. The absorption band $1632 \mathrm{~cm}^{-1}$ was due to carbonyl stretch vibrations in amide linkages and was recognised as amide $\mathrm{I}^{4}$. Bending vibration of N-H bond from primary amines was indicated by $1599 \mathrm{~cm}^{-1}$ (Figure.4). Absorption bands at $1055 \mathrm{~cm}^{-1} \& 1149 \mathrm{~cm}^{-1}$ may represents the $\mathrm{C}-\mathrm{O}-\mathrm{H}$ bending vibrations and $\mathrm{C}-\mathrm{O}$ stretching vibrations due to the proteins and rhamnolipids respectively ${ }^{32}$. Stretching vibrations of $\mathrm{C}-\mathrm{O}-\mathrm{C}$ bond in rhamnose sugar was evidenced by the absorption band at $1022 \mathrm{~cm}^{-1}$ (Figure.4). Wave number $1736 \mathrm{~cm}^{-1}$ indicated the stretching vibration of carbonyl $(\mathrm{C}=\mathrm{O})$ group in rhamnolipids ${ }^{35}$.

$\mathrm{C}-\mathrm{H}$ stretching symmetric and anti-symmetric modes were noticed in the range $2869-2978 \mathrm{~cm}^{-1}$ which represents the aliphatic and aromatic compounds respectively ${ }^{36}$. Remarkably, strong absorption 
band $1368 \mathrm{~cm}^{-1}$ from bending vibrations of carboxylic acid functional group confirmed the presence of rhamnolipids on $\mathrm{AgNPs}^{35}$. The functional group $(\mathrm{C}-\mathrm{H})$ has been identified in the characterization of purified rhamnolipids molecules ${ }^{37}$. FTIR results showed the presence of capping proteins and rhamnolipids on the surface of AgNPs. This could be inferred that proteins along with rhamnolipids might be acting as stabilizing agent (Figure.4). Current results corroborate with our previous findings of stabilizing proteins in biogenic AgNPs ${ }^{22}$. Protein molecules might be attached with AgNPs by cysteine residues which prevent aggregations (Figure.3). FTIR result was also in close agreement with TEM results.

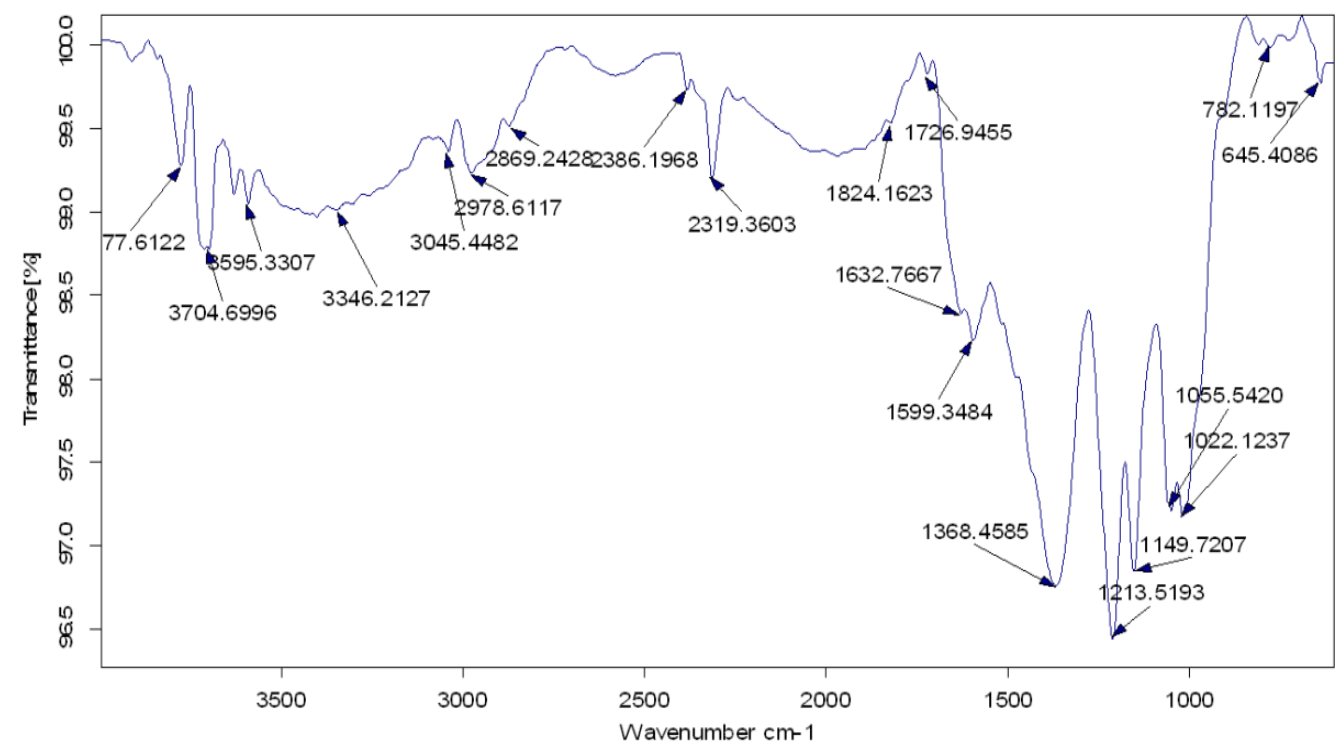

Figure. 4: FTIR spectrum of AgNPs synthesized by Pseudomonas aeruginosa JP1.

\subsection{Possible mechanism of nanoparticle synthesis}

Elucidation of synthesis mechanism was initiated with enzyme characterization by screening the crude extract for nitrate reductase activity. Later, total protein was precipitated out and fractionated by size exclusion chromatography. The fraction with highest specific activity $(5.68 \mathrm{U} / \mathrm{mg})$ was subjected to SDS-PAGE analysis. Molecular size of purified nitrate reductase was $65 \mathrm{kDa}$ (Figure.5), which may belong to respiratory or periplasmic nitrate reductases involved in metal reduction ${ }^{16}$. The molecular size of nitrate reductase was in close range as previously reported $(70 \mathrm{kDa})^{38}$. In order to explore the underlying molecular mechanism of biogenic AgNPs synthesis, purified nitrate reductase was retreated with $\mathrm{AgNO}_{3}$ solution (10 $\mathrm{mM}$ ).Enzymatic reduction of $\mathrm{AgNO}_{3}$ to $\mathrm{AgNPs}$ confirmed the nitrate reductase participation in mechanistic pathway.

Further validation was done by pre-heat treatment of purified nitrate reductase and reacting with $\mathrm{AgNO}_{3}$. Heat inactivated enzyme was unable to reduce the $\mathrm{AgNO}_{3}$ solution in tube $\mathrm{B}$, whereas 
noticeable colour change in tube A can attributed to nitrate reductase activity (Figure.6). Moreover, UV-vis spectrum produced SPR band within 435-450 nm range indicating the AgNPs formation and substantiating the participation of nitrate reductase in biosynthesis pathway (Figure.7). Biogenic AgNPs formulation has been demonstrated by characteristic SPR peaks ${ }^{39}$. PIXE analysis also revealed the AgNPs presence (59679.5ppm) in the reaction mixture (Figure.8). Previously, PIXE technique has been successfully used for the detection and quantification of AgNPs in aqueous food matrices ${ }^{40}$.

Heat treatment inactivated the nitrate reductase (Figure.6) which provided the additional evidence to the mechanistic theory (Figure.9). Hence emphasizing the involvement of nitrate reductase in extracellular AgNPs synthesis as described in the literature ${ }^{41}$. The role of catalytic protein in AgNPs synthesis has been elaborated in our previous study ${ }^{22}$. In another study NADH-dependent nitrate reductase mediated synthesis of AgNPs have been investigated ${ }^{21}$. Previously, Periplasmic nitrate reductases (Nap) and Respiratory nitrate reductases (Nar) have been associated with the biosynthesis of AgNPs ${ }^{16}$. Recently, a similar mechanism was proposed for superoxide-mediated synthesis of AgNPs ${ }^{20}$. Consequently, it can be anticipated that nitrate reductase is a key player in the plausible mechanism of metal transformation into NPs.

Nitrate reductase enables the electron transfer (electron shuttle) from nitrate molecule to the metal ion for NPs formulation (Figure. 9). Stable and size specific nanoscale materials can be synthesized at large scale by optimizing the enzyme physiology. Current findings are in accordance with Jain et al (2011) studies for fungal enzyme mediated synthesis of extracellular AgNPs ${ }^{19}$. The underlying mechanism will provide a breakthrough for synthesizing noble metal nanomaterials with monodispersity and controlled morphologies. Interestingly, microbes have evolved the several mechanisms for metal resistance. The fundamental insight of enzyme-metal interaction is elaborated here which, will enable the biotransformation of toxic heavy metals hence providing the detoxification effect $^{42}$.

Nitrate reductase producing microbes can potentially enhance the efficiency of bioremediation strategies $^{43}$. Metal-microbe interaction and role of secreted enzymes still needs further annotation. A better understanding of microbial transformation pathway at genetic level will leads to develop new genetic tools for accelerating the bioremediation. ${ }^{44}$. Moreover, metal reducing microbes and extracellular electron transfer mechanism may have implications in electro-microbiological applications for renewable energy ${ }^{23}$. 


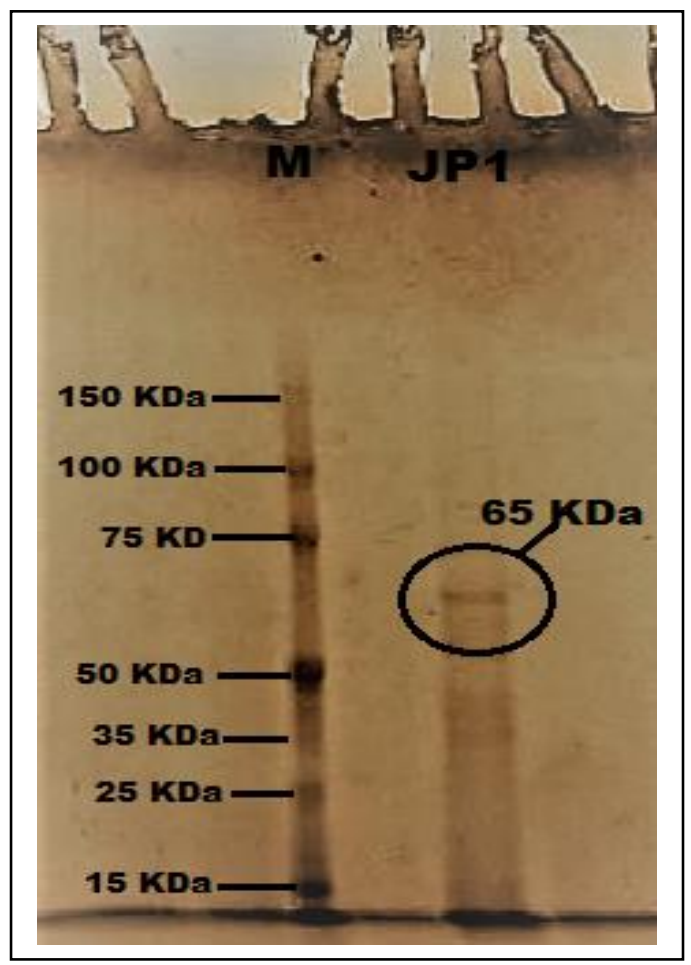

Figure 5: SDS- PAGE of purified nitrate reductase of Pseudomonas aeruginosa JP1.

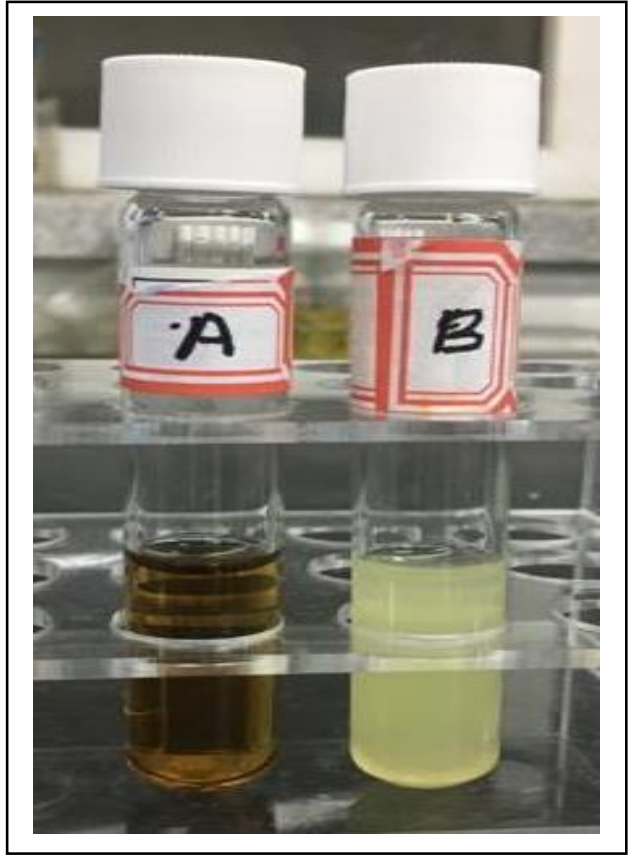

Figure. 6: Tube A showing visible colour change in reaction mixture of purified enzyme and $\mathrm{AgNO}_{3}$ and tube $\mathrm{B}$ shows no colour change by inactivated enzyme and $\mathrm{AgNO}_{3}$. 


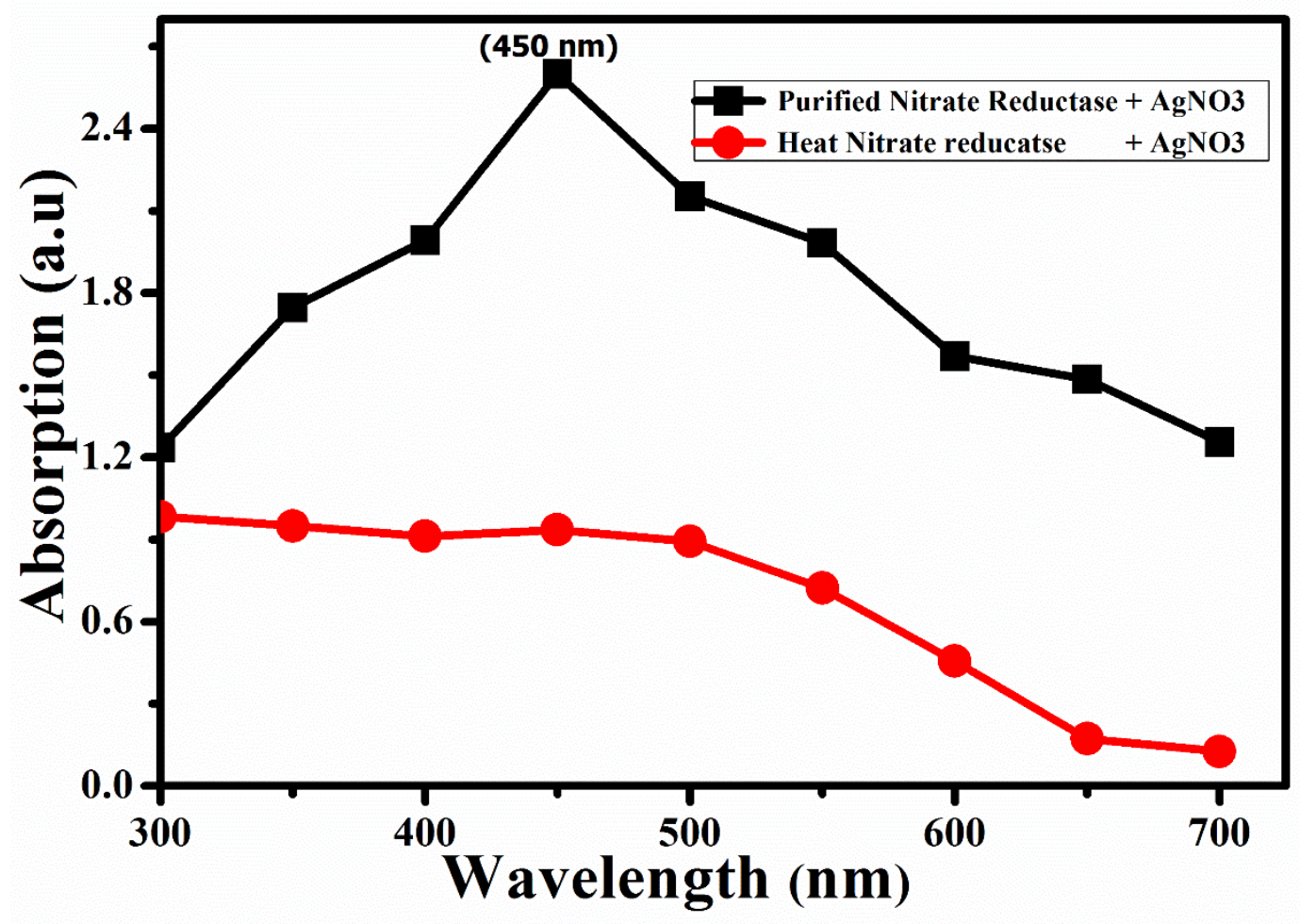

Figure.7 .UV-Visible spectrum of reaction mixtures containing the purified enzyme and AgNO3 solution.

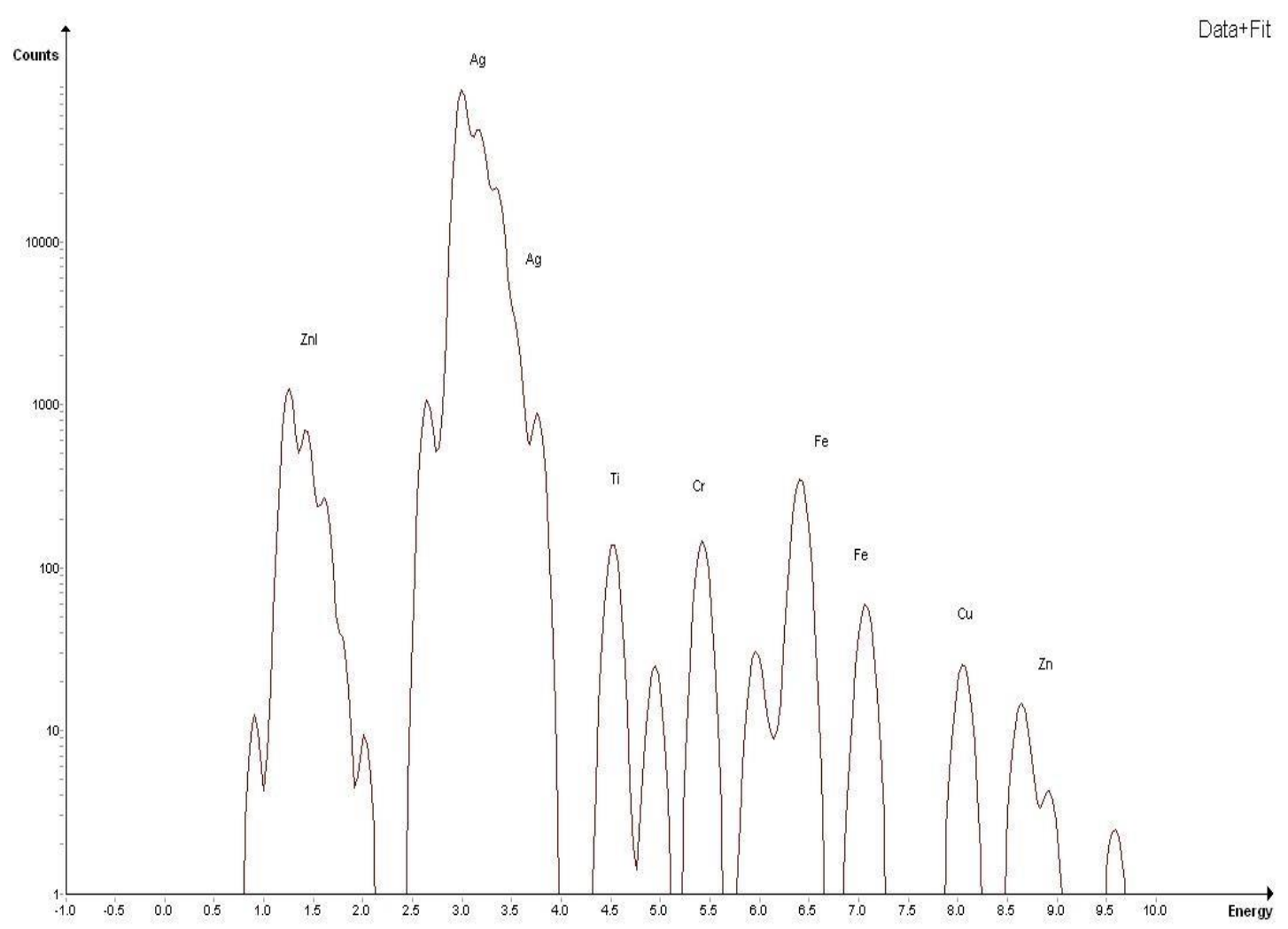

Figure. 8: PIXE Spectrum of AgNPs synthesized by purified nitrate reductase. 


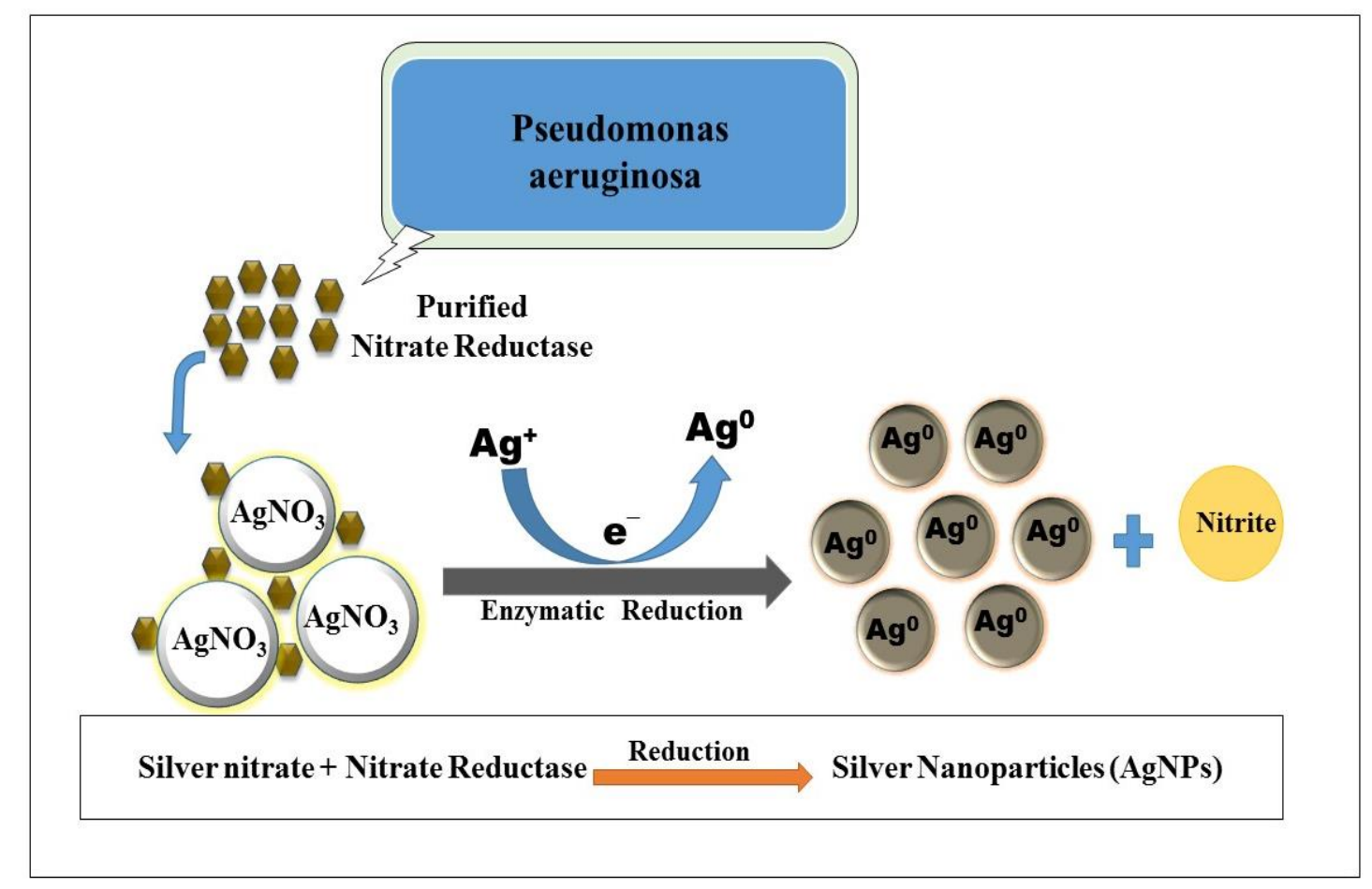

Figure. 9: Possible mechanism for extracellular synthes is of AgNPs

\section{Conclusion}

Microorganisms can reduce $\mathrm{Ag}^{+}$to AgNPs and potentially can serve as nano- factories. Here we reported that nitrate reductase plays a crucial role in extracellular AgNPs synthesis by Pseudomonas aeruginosa JP1. This study is helpful in understanding the mechanism involved green synthesis of AgNPs, which will have many applications regarding the enhanced synthesis of AgNPs with controlled dimensions. Nitrate reductase producing microbes may also have a great implication in electromicrobiology related processes like MFCs for exploring the electron transfer mechanism to electrodes. It is expected that results of present study will also provide a detailed understanding of biomineralization and biotransformation processes and biogeochemical cycles for silver and other heavy metals.

\section{Acknowledgements}

The authors are obliged to the National Centre for Physics, Pakistan and National Institute for Biotechnology and Genetic Engineering (NIBGE), Pakistan for providing technical assistance regarding PIXE and TEM operations respectively. 


\section{References}

1. Patra, S.; Naik, A. N.; Pandey, A. K.; Sen, D.; Mazumder, S.; Goswami, A., Silver nanoparticles stabilized in porous polymer support: A highly active catalytic nanoreactor. Applied Catalysis A: General 2016, $524,214-222$.

2. Nazar, U.; Ali, J.; Ali, Q. u. A.; Ahmad, N. M.; Sarwar, F.; Waseem, H.; Jamil, S. U. U., Improved Antifouling Potential of Polyether Sulfone Polymeric Membrane Containing Silver Nanoparticles; Self-cleaning Membranes. Environmental Technology 2017, (just-accepted), 1-20.

3. Temboury, M. R. C.; Paolucci, V.; Hooley, E. N.; Latterini, L.; Vosch, T., Probing DNA-stabilized fluorescent silver nanocluster spectral heterogeneity by time-correlated single photon counting. Analyst 2016, 141 (1), 123-130.

4. Dutta, D.; Sahoo, A. K.; Chattopadhyay, A.; Ghosh, S. S., Bimetallic silver nanoparticle-gold nanocluster embedded composite nanoparticles for cancer theranostics. Journal of Materials Chemistry B 2016, 4 (4), 793-800.

5. $\quad$ Abbasi,E; Milani, M.; Fekri Aval, S.; Kouhi, M.; Akbarzadeh, A.; Tayefi Nasrabadi, H.; Nikasa, P.; Joo, S. W.; Hanifehpour, Y.; Nejati-Koshki, K., Silver nanoparticles: synthesis methods, bio-applications and properties. Critical reviews in microbiology 2016, 42 (2), 173-180.

6. Mohammadi, S.; Pourseyedi, S.; Amini, A., Green synthesis of silver nanoparticles with a long lasting stability using colloidal solution of cowpea seeds (Vigna sp. L). Journal of Environmental Chemical Engineering 2016, 4 (2), 2023-2032.

7. Reverberi, A.; Kuznetsov, N.; Meshalkin, V.; Salerno, M.; Fabiano, B., Systematical analysis of chemical methods in metal nanoparticles synthesis. Theoretical Foundations ofChemical Engineering 2016, 50 (1), 59-66.

8. da Silva, R. R.; Yang, M.; Choi, S.-I.; Chi, M.; Luo, M.; Zhang, C.; Li, Z.-Y.; Camargo, P. H.; Ribeiro, S. J. L.; Xia, Y., Facile Synthesis of Sub-20 nm Silver Nanowires Through a Bromide-Mediated Polyol Method. ACS nano 2016, 10 (8), 7892-7900.

9. Klaus, T.; Joerger, R.; Olss on, E.; Granqvist, C.-G., Silver-based crystalline nanoparticles, microbially fabricated. Proceedings of the National Academy of Sciences 1999,96 (24), 13611-13614.

10. Jafar Ali, S. Z.; Ali, N., Green Synthesis of Metal nanoparticles by microorganisms; a current prospective. J. Nanoanal 2015,2 (1), 7.

11. Musarrat, J.; Dwivedi, S.; Singh, B. R.; Al-Khedhairy, A. A.; Azam, A.; Naqvi, A., Production of antimicrobial silver nanoparticles in water extracts of the fungus Amylomyces rouxii strain KSU-09.

Bioresource technology 2010,101 (22), 8772-8776.

12. Rengga, W. D. P.; Chafidz, A.; Sudibandriyo, M.; Nasikin, M.; Abasaeed, A. E., Silver nano-particles deposited on bamboo-based activated carbon for removal of formaldehyde. Journal of Environmental Chemical Engineering 2017, 5 (2), 1657-1665.

13. Singh, R.; Shedbalkar, U. U.; Wadhwani, S. A.; Chopade, B. A., Bacteriagenic silver nanoparticles: synthesis, mechanis m, and applications. Applied microbiology and biotechnology 2015,99 (11), 4579-4593.

14. Prabhu, S.; Poulose, E. K., Silver nanoparticles: mechanism of antimicrobial action, synthesis, medical applications, and toxicity effects. International Nano Letters 2012, 2 (1), 1-10.

15. Bramhachari, P.; Nagaraju, G. P., Extracellular Polysaccharide Production by Bacteria as a Mechanis $m$ of Toxic Heavy Metal Biosorption and Biosequestration in the Marine Environment. In Marine Pollution and Microbial Remediation, Springer: 2017; pp 67-85.

16. Wing-ShanáLin, I., Biosynthes is of silver nanoparticles from silver (i) reduction by the periplasmic nitrate reductase c-type cytochrome subunit NapC in a silver-resistant E. ácoli. Chemical Science 2014, 5 (8), 3144-3150.

17. Durán, N.; Marcato, P. D.; Alves, O. L.; De Souza, G. I.; Esposito, E., Mechanistic aspects of biosynthesis of silver nanoparticles by several Fusarium oxysporum strains. Journal of nanobiotechnology 2005, $3(1), 1$.

18. Shahverdi, A. R.; Minaeian, S.; Shahverdi, H. R.; Jamalifar, H.; Nohi, A.-A., Rapid synthesis of silver nanoparticles using culture supernatants of Enterobacteria: a novel biological approach. Process Biochemistry 2007, 42 (5), 919-923.

19. Jain, N.; Bhargava, A.; Majumdar, S.; Tarafdar, J.; Panwar, J., Extracellular biosynthesis and characterization of silver nanoparticles using Aspergillus flavus NJP08: a mechanism perspective. Nanoscale 2011,3 (2), 635-641.

20. Yin, Y.; Yang, X.; Hu, L.; Tan, Z.; Zhao, L.; Zhang, Z.; Liu, J.; Jiang, G., Superoxide-Mediated Extracellular Biosynthesis of Silver Nanoparticles by the Fungus Fusarium oxysporum. Environmental Science \& Technology Letters 2016,3 (4), 160-165. 
21. Talekar, S.; Joshi, A.; Chougle, R.; Nakhe, A.; Bhojwani, R., Immobilized enzyme mediated synthesis of silver nanoparticles using cross-linked enzyme aggregates (CLEAs) of NADH-dependent nitrate reductase. Nano-Structures \& Nano-Objects 2016, 6, 23-33.

22. Ali, J.; Hameed, A.; Ahmed, S.; Ali, M. I.; Zainab, S.; Ali, N., Role of catalytic protein and stabilizing agents in transformation of Ag ions to nanoparticles by Pseudomonas aeruginosa. IET Nanobiotechnology 2016. 23. Jackson, M.; Mantsch, H. H., The use and misuse of FTIR spectroscopy in the determination of protein structure. Critical reviews in biochemistry and molecular biology 1995, 30 (2), 95-120.

24. Kruger, N. J., The Bradford method for protein quantitation. Basic protein and peptide protocols 1994, 9-15.

25. Saifuddin, N.; Wong, C.; Yasumira, A., Rapid biosynthes is of silver nanoparticles using culture supernatant of bacteria with microwave irradiation. Journal of Chemistry 2009, 6 (1), 61-70.

26. Bollag, D. M., Gel-Filtration Chromotography. Peptide Analysis Protocols 1994, 1-9.

27. Laemmli, U.; Favre, M., SDS Polyacrylamide gel electrophoresis. Nature 1970, 227, 680-682.

28. Johansson, S. A.; Campbell, J. L., PIXE: A novel technique for elemental analysis. 1988.

29. Campbell, J., GUPIX and GUPIXWIN homepage [Internet]. Available from the website: $\underline{\text { http://pixe }}$. physics. uoguelph. ca/gupix/main 2005.

30. Saha, N.; Trivedi, P.; Gupta, S. D., Surface Plasmon Resonance (SPR) Based Optimization of Biosynthesis of Silver Nanoparticles from Rhizome Extract of Curculigo orchioides Gaertn. and Its Antioxidant Potential. Journal of Cluster Science 2016, 27 (6), 1893-1912.

31. Das, V. L.; Thomas, R.; Varghese, R. T.; Soniya, E.; Mathew, J.; Radhakrishnan, E., Extracellular synthesis of silver nanoparticles by the Bacillus strain CS 11 isolated from industrialized area. 3 Biotech 2014, 4 (2), 121-126.

32. Kumar, C. G.; Mamidyala, S. K., Extracellular synthesis of silver nanoparticles using culture supernatant of Pseudomonas aeruginosa. Colloids and Surfaces B: Biointerfaces 2011, 84 (2), $462-466$. 33. Fayaz, A. M.; Balaji, K.; Girilal, M.; Yadav, R.; Kalaichelvan, P. T.; Venketesan, R., Biogenic synthesis of silver nanoparticles and their synergistic effect with antibiotics: a study against gram-positive and gram-negative bacteria. Nanomedicine: Nanotechnology, Biology and Medicine 2010,6 (1), 103-109.

34. Zaki, S.; El Kady, M.; Abd-El-Haleem, D., Biosynthesis and structural characterization of silver nanoparticles from bacterial isolates. Materials research bulletin 2011,46 (10), 1571-1576.

35. Mulligan, C. N.; Sharma, S. K.; Mudhoo, A., Biosurfactants: research trends and applications. CRC Press: 2014.

36. Kumar, C. G.; Mamidyala, S. K.; Das, B.; Sridhar, B.; Devi, G. S.; Karuna, M. S., Synthesis of biosurfactant-based silver nanoparticles with purified rhamnolipids isolated from Pseudomonas aeruginosa BS161R. J Microbiol Biotechnol 2010,20, 1061-1068.

37. Soltani Dashtbozorg, S.; Kohl, J.; Ju, L.-K., Rhamnolipid Adsorption in Soil: Factors, Unique Features, and Considerations for Use as Green Antizoosporic Agents. Journal of agricultural and food chemistry 2016, 64 (17), 3330-3337.

38. Kathiresan, K.; Manivannan, S.; Nabeel, M.; Dhivya, B., Studies on silver nanoparticles synthesized by a marine fungus, Penicillium fellutanum isolated from coastalmangrove sediment. Colloids and surfaces B: Biointerfaces 2009, 71 (1), 133-137.

39. San Keskin, N. O.; Kiliç, N. K.; Dönmez, G.; Tekinay, T., Green Synthesis of Silver Nanoparticles Using Cyanobacteria and Evaluation of Their Photocatalytic and Antimicrobial Activity. Journal of Nano Research 2016, 40, 120.

40. Lozano, O.; Mejia, J.; Tabarrant, T.; Masereel, B.; Dogné, J.-M.; Toussaint, O.; Lucas, S., Quantification of nanoparticles in aqueous food matrices using particle-induced X-ray emission. Analytical and bioanalytical chemistry 2012,403 (10), 2835-2841.

41. El- Baz, A. F.; El- Batal, A. I.; Abomosalam, F. M.; Tayel, A. A.; Shetaia, Y. M.; Yang, S. T., Extracellular biosynthesis of anti- Candida silver nanoparticles using Monascus purpureus .Journal ofbasic microbiology 2015.

42. Liu, S.-H.; Zeng, G.-M.; Niu, Q.-Y.; Liu, Y.; Zhou, L.; Jiang, L.-H.; Tan, X.-f.; Xu, P.; Zhang, C.; Cheng, M., Bioremediation mechanisms of combined pollution of PAHs and heavy metals by bacteria and fungi: A mini review. Bioresource Technology 2016.

43. Noor Afifah, F.; Hajar, S.; Rasdi, M.; Essam, A. M.; Hasbi, M.; Rahim, A., Bioremediation of Disposed X-Ray Film For Enzymes Production. Global Journal of Advanced Research (GJAR) 2016, 3 (2), 101106.

44. Kang, F.; Qu, X.; Alvarez, P. J.; Zhu, D., Extracellular Saccharide-Mediated Reduction of Au3+ to Gold Nanoparticles: New Insights for Heavy Metals Biomineralization on Microbial Surfaces. Environmental Science \& Technology 2017. 